\title{
Influence of Fluid on Seal and Assembly of Pipeline Fittings Based on the Multiscale Finite Element Model
}

\author{
Yangyang Yan (iD) and Yaping Fan \\ Facility Horticulture Laboratory of Universities in Shandong, Weifang University of Science and Technology, \\ Weifang 262700, China \\ Correspondence should be addressed to Yangyang Yan; yyysar@163.com
}

Received 26 December 2019; Revised 29 March 2020; Accepted 17 April 2020; Published 7 May 2020

Academic Editor: Quanmin Zhu

Copyright (c) 2020 Yangyang Yan and Yaping Fan. This is an open access article distributed under the Creative Commons Attribution License, which permits unrestricted use, distribution, and reproduction in any medium, provided the original work is properly cited.

\begin{abstract}
Pipeline fittings with ferrules are applied to connect sections of hydraulic pipelines in aircraft, and their reliability and stability are essential. This paper aims at investigating the influence of internal fluid on the sealing characteristics of pipeline fittings by employing the multiscale model. Changes in the sealing characteristics induced by the fluid pressure switch are studied, and the assembly method under the internal fluid is also explored. The calculated results show that the multiscale model can accurately reflect the changes in the sealing area, and the high-pressure fluid can enhance the sealing reliability. Compared with the contact area, the fluid pressure exerts a greater influence on the change in the area of the high-stress zone. Furthermore, the unrestored sealing area enlarges with the increased maximum fluid pressure, and the change in the area of the high-stress zone is significantly larger than that in the contact area. Moreover, the optimum assembly position of ferrule decreases with the increase in fluid pressure, thus achieving the excellent sealing characteristics.
\end{abstract}

\section{Introduction}

It is crucial to design a hydraulic system with high pressure, high power, and lightweight [1]. Increasing the system fluid pressure provides an effective way to reduce system weight. However, the high-pressure fluid can cause radial deformation of the pipeline as cyclical changes, which leads to the formation of a cyclic load on the pipeline wall. In addition, it results in a sudden change of the fluid state to form a hydraulic shock when the hydraulic system starts, stops, or performs a large maneuver. These loads can influence the seal of pipeline fittings where fluid leakage often occurs. Therefore, the sealing characteristics of pipeline fittings are influenced by the high-pressure fluid, and it is crucial to investigate the sealing characteristic change of pipeline fittings caused by the fluid pressure switching.

A lot of achievements have been made on the seal of pipeline fittings. For example, Xia [2] discussed the classification and application of pipeline fittings in detail. Mihsein and Seymour [3] simulated the assembly state of pipeline fittings based on finite element simulation and verified the accuracy of the simulation results by X-ray photography technology. Yan et al. [4] studied the sealing principle of pipeline fittings and proposed an assembly approach of pipeline fittings. Based on the previous research, most strategies are about dealing with the sealing state of pipeline fittings.

Currently, few reports focus on the influence of internal fluid on the sealing characteristics of pipeline fittings. However, there are numerous studies associated with the fluid pressure affecting the sealing characteristics of other sealing structure. Li et al. [5] analyzed the influence of working pressure on the contact state and stress of the mechanical seal face. Metcalfe et al. [6] introduced three types of high-pressure water full lubrication tests for sealing parts of large-scale circulating pumps and conducted leakage prediction and deflection analysis. Willenborg et al. [7] employed an experimental method to determine the effect of the Reynolds number and pressure ratio on operating features of a stepped labyrinth seal. Li et al. [8] determined the 
influence of the pressure ratio and sealing clearance on the fluid leakage characteristics in a honeycomb stepped labyrinth seal. Gibson et al. [9] studied the impact of the interaction between pressure fields from an upstream vane row and downstream blade row on hot gas ingress for enginerealistic rim seal geometries. Generally, most previous studies used a macromodel of the sealing structure to investigate the influence of the fluid pressure on the sealing characteristics, which exhibits numerous limitations.

Recently, the research about the sealing characteristics is mainly based on the contact model with rough surfaces. Kucharski and Starzynski [10] investigated the contact characteristics between rough surfaces with a rigid flat plane based on experimental and numerical methods. Zhao et al. [11] studied elastic-plastic contact between rough surfaces through the Hertz contact theory. An et al. [12] used a novel method of spectral interpolation for the elastic-plastic modeling of rough surface contact. Guo et al. [13] established a theoretical contact model of revolute joints with a planar rough surface. Moreover, in other reports [14-18], the rough surface contact model considering other different influencing factors is mainly discussed. Therefore, the contact model with rough surfaces is primarily based on a simple principle model, while it shows some limitations to analyze the sealing characteristics. Due to the limitations of the contact model with a rough surface, the multiscale model gradually becomes the most appropriate strategy to study the sealing characteristics. For instance, Wenk et al. [19] analyzed the contact and deformation characteristics of the radial lip seal structure by a three-dimensional (3D) multiscale model. Pérez-Ràfols et al. [20] developed contact properties between rough surfaces and the fluid flow between rough voids based on rough metal surfaces. Jia et al. [21] predicted the pumping rate of the rotary lip seal from the air end to the fluid end using the multiscale finite element model. Zhang et al. [22] employed a 3D model to calculate the passage and rate of leakage between rough surfaces with the classical method. Thatte and Salant [23] established a multiscale model to study the impact of viscoelasticity on the sealing behavior of a hydraulic piston rod. Marie et al. [24] modified the multiscale contact model based on the leakage rate of the seal structure obtained from the test to achieve a more practical and applied multiscale calculation model. Further, the accuracy of the model was verified by the real test simulation.

As an important component of fluid seal and pipeline connection, the sealing characteristics of pipeline fittings are very important to pipeline system. During the service period, the contact sealing area and contact stress distribution of pipeline fittings are important indicators to measure the sealing characteristics of pipeline fittings. Effective fluid seal can be ensured only with sufficient sealing area and uniform contact stress distribution. In addition, due to the action of high-pressure fluid, high-stress area (plastic deformation area) will appear in the sealing area, which is the key line of defense to ensure fluid sealing. Therefore, based on the measured roughness data of the sealing area of pipeline fittings, this paper establishes the multiscale model of pipeline fittings to calculate and analyze the change of the sealing characteristics of pipeline fittings more accurately. Through the macro- and microdeformation of the sealing area and the contact stress distribution of pipeline fittings, the influence of high-pressure fluid on the sealing characteristics of pipeline fittings is analyzed in detail. In addition, the influence of the internal fluid on the sealing characteristics and the sealing change of fittings after fluid pressure switch are investigated. Meanwhile, the assembly method of the pipeline fittings can be obtained under the action of the high-pressure fluid.

\section{Multiscale Modeling Method considering Fluid}

As shown in Figure 1, pipeline fittings show a symmetrical morphology, containing nuts, fitting body, back ferrule, and front ferrule. The metal-to-metal seal with certain prestress is realized by both ferrules penetrating the outer diameter of pipeline. The surface characteristics of pipeline structure have a significant impact on the stress state of surfaces. In this study, the multiscale modeling of pipeline fittings is conducted using the fluid pressure as the main influencing factor. The model takes the actual surface features of the sealing zone and analyzes the sealing state between pipeline fittings and pipeline under high-pressure fluid, which provides a mechanistic explanation for the sealing characteristics change of pipeline fittings.

This paper and reference [25] belong to a part of systematic research on the sealing characteristics of pipeline fittings, and the differences and innovations are as follows: (1) Based on the multiscale modeling method of pipeline fittings in reference [25], this paper analyzes the influence of high-pressure fluid load on the sealing characteristics of pipeline fittings in depth and in detail through more accurate quantification and analysis methods of sealing characteristics of different pipeline fitting materials. It can be concluded that the high-pressure fluid has a significant impact on the contact state of the sealing area of pipeline fittings, and highpressure fluid can improve the sealing reliability of pipeline fittings. (2) Considering the tangent modulus of the material of pipeline fittings, the sealing characteristics of pipeline fittings are analyzed in the process of pressure rise and pressure drop, and the leakage mechanism of pipeline fittings is obtained after the change from high pressure to low pressure. (3) As the high-pressure pulsating fluid load seriously affects the sealing characteristics of pipeline fittings in service, the more scientific and effective assembly method of pipeline fittings is achieved under the action of highpressure fluid.

2.1. Multiscale Model Generation. Based on the Zygo test of ferrule and pipeline surface topography data, a multiscale model of pipeline fittings is established in this paper, which can simultaneously reflect the microscopic and macroscopic dimensions of the sealing zone of pipeline fittings. Figure 2 presents the surface topography data of ferrule and pipeline. The peak-to-valley value of the corrugation fluctuates within a range of $\pm 5 \mu \mathrm{m}$ and $\pm 2 \mu \mathrm{m}$. Due to the optical interference, 


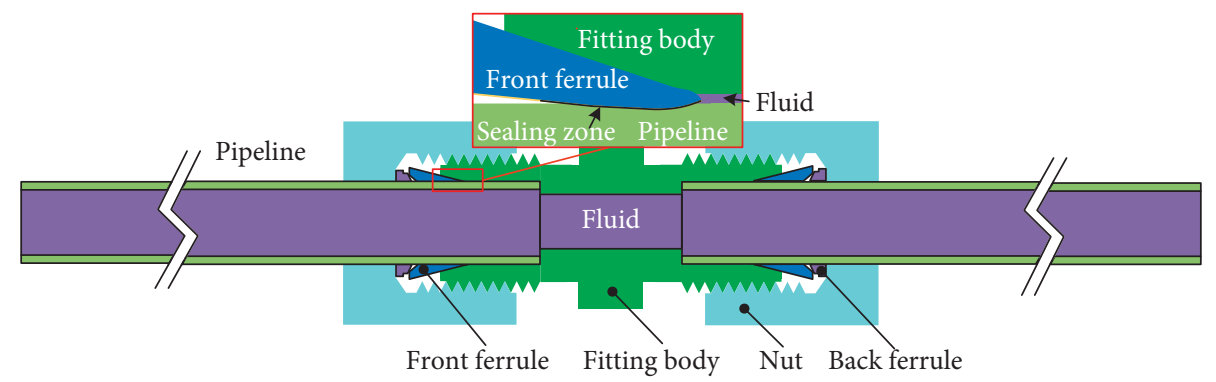

FIgURE 1: Structure of pipeline fittings.
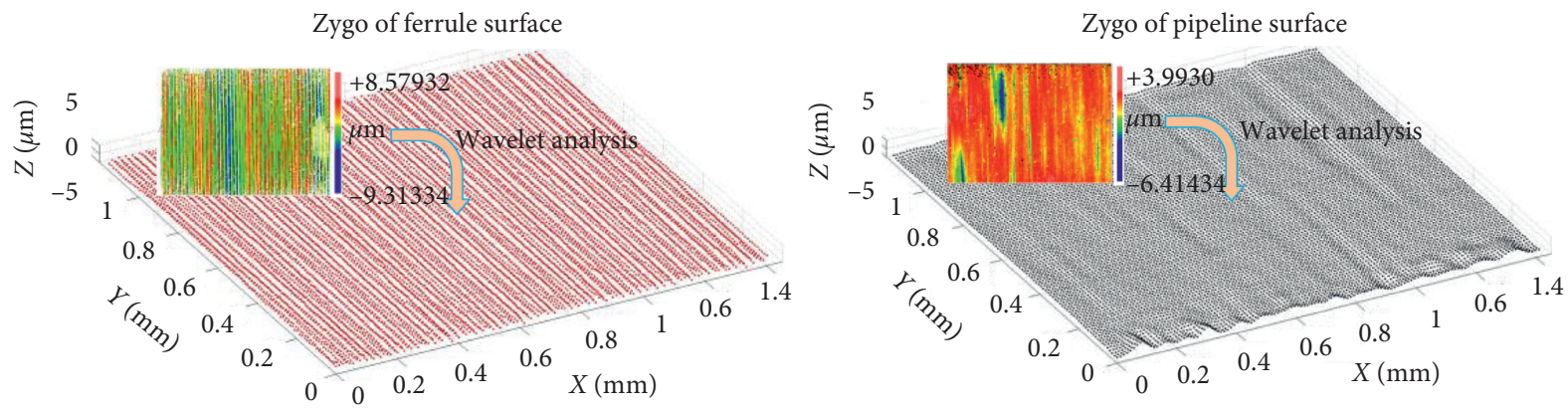

FIGURE 2: Zygo test and surface morphology of ferrule and pipeline after wavelet analysis.

it is necessary to filter the interference signal by conducting wavelet analysis to obtain the postprocessing result, which can be directly used in the model. Figure 2 reveals the results of the wavelet analysis, which is required for subsequent reverse modeling.

Based on the reverse modeling process, the multiscale model of the pipeline fittings is constructed. It is imported into the finite element software ANSYS and meshed with SOLID186. The mesh size is $0.2 \mu \mathrm{m}$. Figure 3 presents the multiscale finite element models of the ferrule and pipeline. In the finite element calculation, ferrule, pipeline, and fitting body are all made of stainless steel. Table 1 lists the material characteristics data.

\subsection{Boundary Conditions of the Multiscale Model. After} pipeline fittings are tightened, the pipeline, ferrule, and fitting body are closely attached together, and the fluid pressure in the pipeline directly acts on the inner surface of the pipeline. Besides, the internal pressure action form mainly represents the inner surface of pipeline. The axial fluid pressure force is ignored in the radial expansion extrusion. Meanwhile, the fluid pressure force is transmitted from the pipeline to the ferrule and the fitting body. Due to the uniform fluid pressure and the stability of the finite element calculation, the fluid pressure is applied on the inner pipeline surface, thereby realizing the application of the high-pressure fluid load.

As the multiscale model of pipeline fittings is based on a certain sealing area in the circumferential direction of pipeline fittings, the axisymmetric boundary condition is adopted in the multiscale model. Namely, the nodes perpendicular to the circumferential plane are fixed to constrain the circumferential displacement of the model, and the

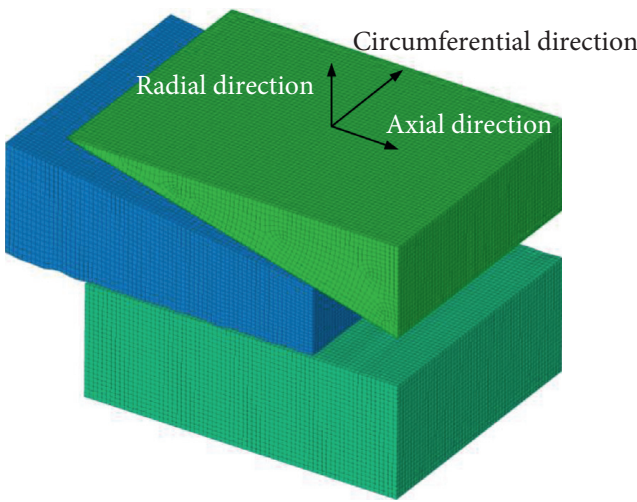

Figure 3: Multiscale model of pipeline fittings.

TABLE. 1: Material properties of pipeline fittings.

\begin{tabular}{lccc}
\hline & Symbol & Unit & Value \\
\hline Density of stainless steel & $\rho$ & $\mathrm{kg} / \mathrm{m}^{3}$ & 7930 \\
Young's modulus of stainless steel & $E$ & $\mathrm{~Pa}$ & $1.95 \times 10^{11}$ \\
Poisson's ratio of stainless steel & $\mu$ & & 0.247 \\
Yield strength of stainless steel & & $\mathrm{MPa}$ & 208 \\
\hline
\end{tabular}

nodes perpendicular to the radial plane are fixed to constrain the radial displacement of the model. In addition, the same constraint method is used for the axial face of ferrule. As illustrated in Figure 4, the multiscale model without internal fluid is used to simulate the tightening process of pipeline fittings by applying displacement on ferrule. When the ferrule is tightened to a certain position, the common pressure value of the hydraulic system is applied on the inner surface of the pipeline. Without considering the fluid structure coupling, the corresponding fluid pressure load is 
applied on all nodes of the inner surface of the pipeline to simulate the effect of the fluid pressure on pipeline fittings. Figure 4 shows the fluid pressure application position, and Figure 3 presents the multiscale finite element model of pipeline fittings. Table 1 displays the material of pipeline fittings.

\section{Results and Discussion}

3.1. Sealing Analysis of Fittings during Assembly. Figure 5(a) shows the stress distribution of pipeline fittings during the assembly process. Because the front end of ferrule can be regarded as line-to-plane contact with fitting body, the high-stress zone is first generated at this position. With the continuous development of elastic-plastic deformation, the high-stress zone is gradually expanded to the rear of ferrule, as shown in Figure 5(a) (A). When the expansion velocity of the wedge space is smaller than the feeding velocity of ferrule, the entire ferrule undergoes buckling deformation, and the high-stress zone moves in the stress concentration induced by buckling, as shown in Figure 5(b) (B). When the ferrule reaches the position shown in Figure 5(c) (C), the front end of ferrule is detached from the wedge action, and the buckling deformation is gradually relieved and disappeared. In addition, the high-stress zone is distributed near the contact surface again. The above results ensure the close bonding between components and the effective seal of fittings.

Figure 5(b) shows the stress state on the pipeline surface during the assembly process. The equivalent stress zone on the pipeline surface is continuously banded. In the initial stage of the assembly process, the middle part of the pipeline surface is always in a high-stress state. When buckling occurs, the stress reaches the maximum value of the whole process. However, it is not the main area of the pipeline seal, and the sealing stress value is not extremely high. When the front end of ferrule is separated from the wedge-shaped surface, its buckling state disappears. The front end of the ferrule-pipeline interface reaches a favorable stress state, and the microconvex bodies are fully fused. As a result, a favorable sealing state of pipeline fittings is realized.

Figure 5(c) shows the stress distribution on the lower surface of ferrule. Due to the buckling deformation of ferrule during the assembly process, the stress does not exhibit a regular banded distribution, indicating the uneven contact degree and poor seal. When the buckling state of ferrule disappears, a regular banded stress distribution appears in the middle of ferrule. As the main sealing area, the front area possess a high-stress value and large stress coverage area. At a specified assembly position, the deformation can be concentrated on the front area, which leads to obvious elastic deformation or even plastic deformation of local positions. Given that the convex bodies between contact surfaces fuse in large areas, pipeline fittings can achieve a desirable sealing state.

Figure 6 demonstrates the change rule of the sealing characteristics of pipeline fittings. Figure 6(a) shows the sealing change rule of the effective sealing area occupied by the contact area of pipeline surface during the assembly process. Obviously, the axial displacement of ferrule and the contact area rate are positively correlated. In addition, in order to show the accuracy of the multiscale model, the results are compared by the smooth surface model and the rough surface model, as shown in Figure 6(b). Compared with the smooth surface model, the rough surface model can reflect the contact sealing state more truly, which can significantly improve the calculation accuracy of sealing characteristics of pipeline fittings.

In the analysis of the sealing characteristics of pipeline fittings, the sealing area and contact stress distribution of pipeline fittings should also be considered. The pipeline fittings should not only reach a certain sealing area, but also have a uniform contact stress distribution state. Therefore, the microconvex bodies on the rough surface of pipeline fittings are in the best contact state after being fully optimized. For example, when the axial displacement of ferrule reaches $0.338 \mathrm{~mm}$, all parts of the pipeline fittings are in a state of high stress after the tightening process of pipeline fittings, which optimizes the microconvex bodies on the sealing surface of pipeline fittings. Under the uniform high stress, the sealing surface can better realize the effective sealing of the high-pressure fluid in the pipeline.

\subsection{Sealing State Analysis}

3.2.1. Sealing State under High-Pressure Fluid. The hydraulic pipeline is often under the action of the high-pressure fluid. According to the results of the assembly process of pipeline fittings, the typical position of ferrule is selected as the initial stress and deformation state for analyzing the effect of highpressure fluid. To analyze the influence of high-pressure fluid on the sealing state of pipeline fittings, different fluid pressures of $7,14,21,28,35$, and $56 \mathrm{MPa}$ are employed on the inner surface of pipeline, respectively.

According to the change in the contact sealing state of pipeline fittings during the assembly process, the contact state with axial displacement of ferrule of $0.23 \mathrm{~mm}$ is selected as the initial assembly state. The stress distribution of pipeline fittings under different fluid pressures is shown in Figure $7(\mathrm{a})$. This shows that, under the action of lower pressure, the entire pipeline fitting assembly does not undergo significant elastoplastic deformation. With increasing fluid pressure, the high-stress area increases, and the deformation energy is mostly absorbed by pipeline and ferrule. Accordingly, there is no significant stress state change between ferrule and fitting body. When the pressure reaches $21 \mathrm{MPa}$, the area of the high-stress zone and the maximum stress value are further increased, while the degree of change in stress state between ferrule and fitting body slightly decreases. When the pressure increases to $56 \mathrm{MPa}$, the contact area is in a peak stress state. Due to the limitation of material mechanical properties, the maximum stress value stops to rise with the increased fluid pressure in a limited range. The stress state between ferrule and fitting body significantly changes. Moreover, the stress change between ferrule and fitting body also reaches the final state because of the absorption deformation energy of ferrule, and the pipeline 


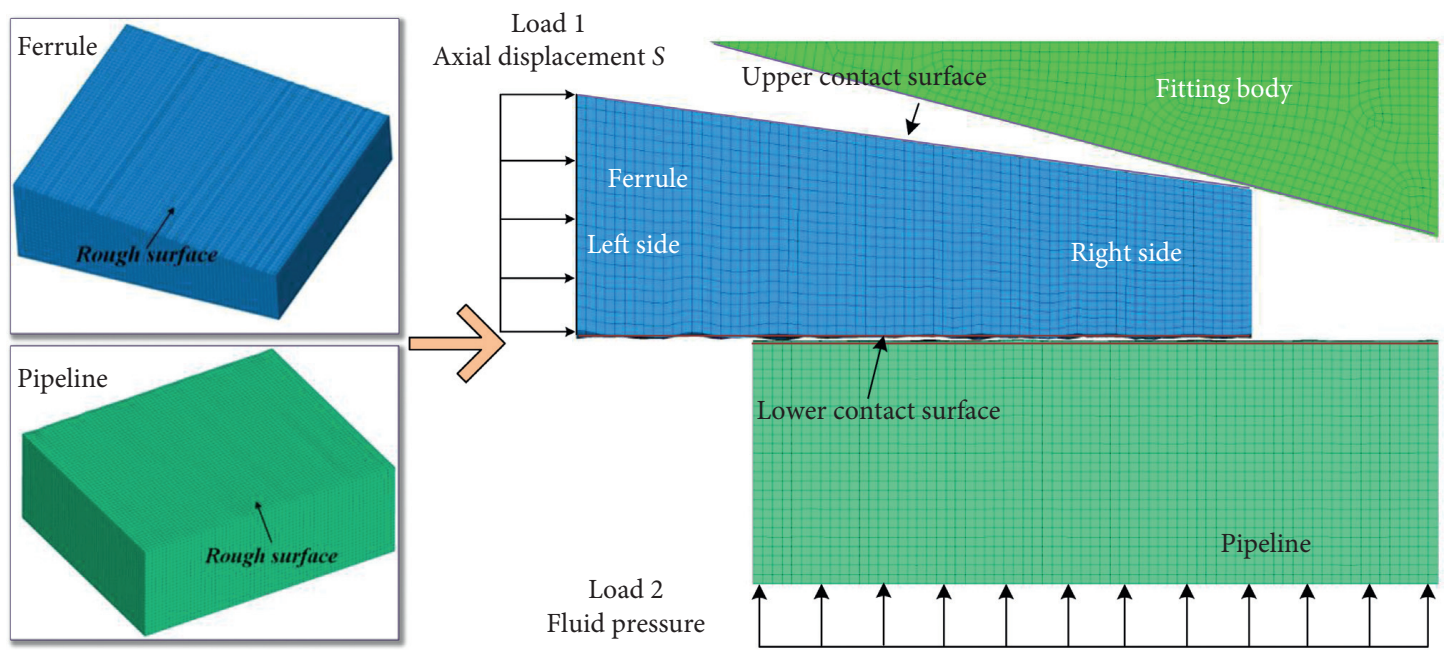

FIgURE 4: Multiscale model of pipeline fittings with the fluid pressure.

reaches a saturation state. Obviously, with the increasing fluid pressure, there is an obvious high-stress area between ferrule and fitting body, and ferrule and pipeline. Besides, the high-stress area and the maximum stress value continuously increase. The greater the contact stress on the same contact area, the better the sealing characteristics. The high fluid pressure will enhance the sealing state of pipeline fittings.

To further verify the sealing state, the stress distribution of the pipeline surface is studied. According to Figure 7(b), with the increasing fluid pressure, the high-stress area on the pipeline surface increases gradually, and the coverage area and peak stress continuously increase. When the pressure reaches $28 \mathrm{MPa}$, the band stress distribution is obvious, and the peak stress is located in the middle of each band. With the increased pressure, the peak stress region expands from the point to the line, and it is perpendicular to the axial direction. The formation of a high-stress band group results in numerous sealing lines between ferrule and pipeline, which can absorb fluid pressure energy step by step and effectively prevent the leakage of fluid.

Figure 7(c) shows the stress distribution on the ferrule surface (which fits the pipeline) under different fluid pressures. Corresponding to Figure 7(b), the stress distribution of the ferrule surface gradually presents a banded distribution law with the increased fluid pressure, and the area of high-stress zone continuously expands. In the effective sealing area of the ferrule surface, the high-stress band is originally located on the left side. When the fluid pressure increases, the high-stress band gradually moves to the right, and the main high-stress sealing band is finally generated on the right side. Similar to the stress distribution on the pipeline surface, the deformation of the microconvex bodies can be concentrated on here, which leads to the formation of the main line of leakage. If the front-end sealing stress is released, the back end can be elastically deformed to a certain extent. Energy supplementation shows that high fluid pressure increases the sealing effectiveness and reliability of pipeline fittings.

\subsubsection{Sealing State under Different Assembly Positions.}

The above research reveals the influence of high-pressure fluid on the sealing state of pipeline fittings when the axial displacement of ferrule is $0.23 \mathrm{~mm}$. Because the assembly position affects the seal of pipeline fittings during the service state, the high-pressure fluid should be considered to explore the optimal axial assembly position, which could ensure the sealing reliability of pipeline fittings.

An internal pressure value is used as the input variable of the multiscale model, and the analysis and calculation of the sealing state of pipeline fittings under different axial assembly positions are conducted. The fluid pressure of $21 \mathrm{MPa}$ is input into the multiscale model, and the axial assembly position of ferrule is changed, which is $S=0.23$, 0.286 , and $0.338 \mathrm{~mm}$, respectively. The stress distribution of pipeline fittings is obtained in Figure 8. In Figure 8(a), the different stress states of pipeline fittings are shown under different axial assembly positions. The general trend is that when the peak value of the high-stress zone increases, the high-stress range increases. Meanwhile, comparing the results of $S=0.23,0.286$, and $0.338 \mathrm{~mm}$, the deformation on the surface of each component can move forward under the high-pressure fluid. When the ferrule is located at the assembly position where the buckling state has just disappeared $(S=0.338 \mathrm{~mm})$, a high energy set is generated at the front end of the ferrule-pipeline contact. In the middle region, the micro-elastic-plastic deformation of the material is obvious, and the convergence of microconvex bodies between contact surfaces increases. Therefore, it can ensure the reliability of the seal under harsh working conditions (such as strong and lasting vibrations).

This study analyzes the stress distribution on the pipeline surface under high-pressure fluid and the development law of the stress state along with the axial displacement of ferrule. A radial observation and analysis angle is applied to verify the above theory. Under the influence of fluid pressure, when the ferrule moves toward the final assembly position, the high-stress zone first forms in the middle of the sealing zone on the pipeline 


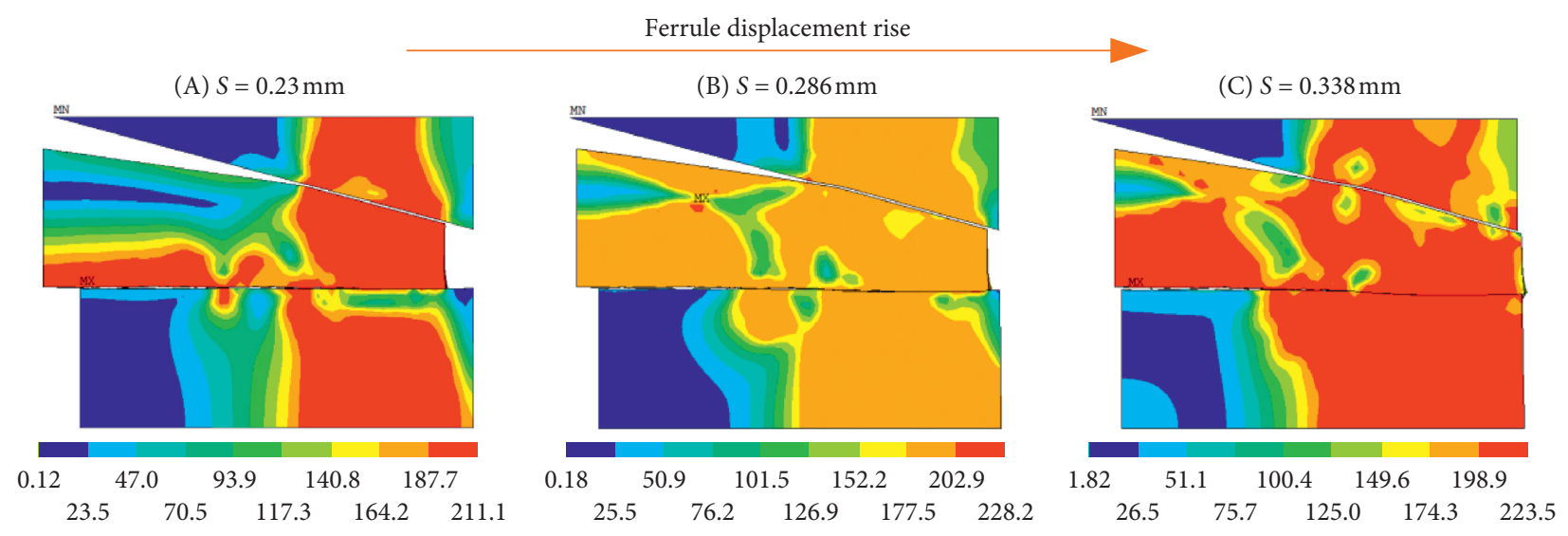

(a)

(A) $S=0.23 \mathrm{~mm}$

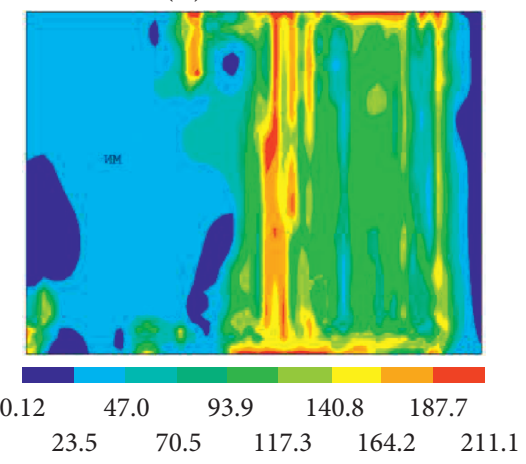

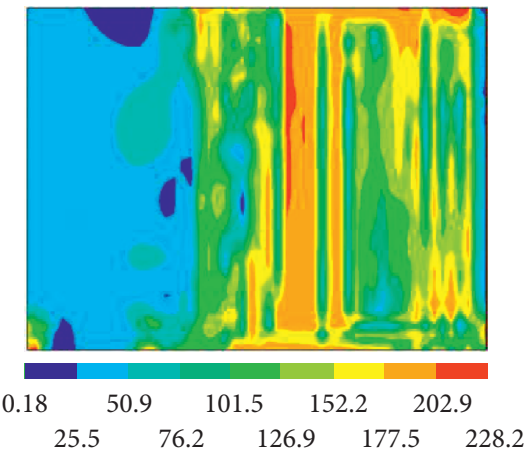

(b)
(C) $S=0.338 \mathrm{~mm}$

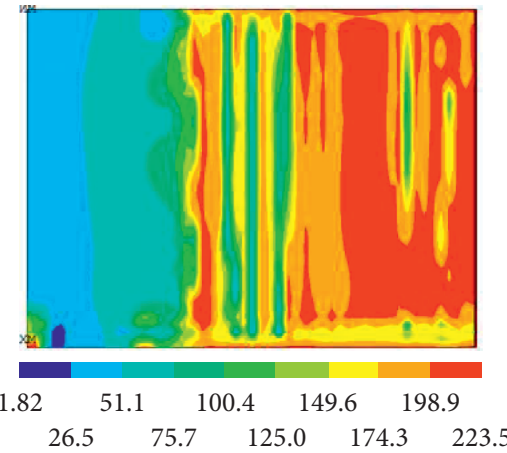

Ferrule displacement rise

(A) $S=0.23 \mathrm{~mm}$

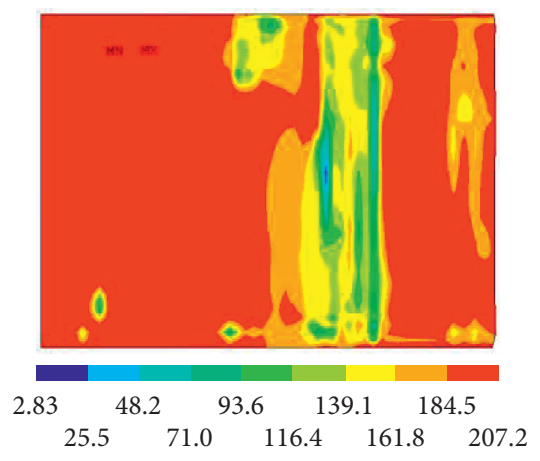

(B) $S=0.286 \mathrm{~mm}$

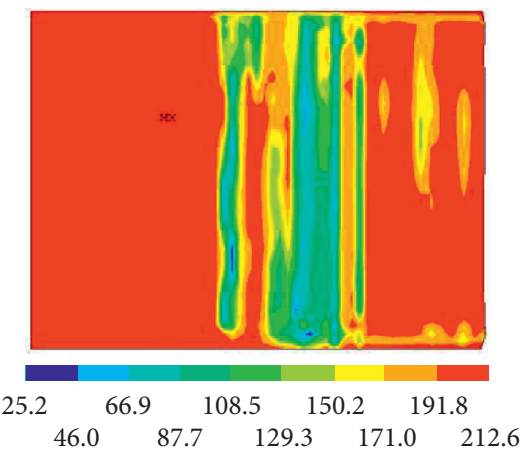

(C) $S=0.338 \mathrm{~mm}$

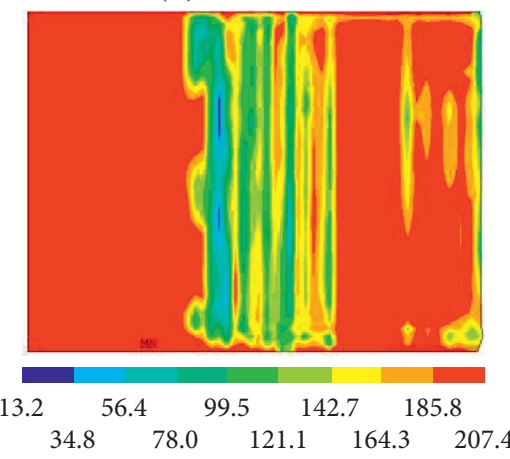

(c)

FIGURE 5: Sealing analysis of pipeline fittings during assembly. (a) Seal state of fittings. (b) Stress distribution of pipeline surface. (c) Stress distribution of ferrule surface.

surface. When the ferrule moves forward, the high-stress zone expands forward and backward. Due to the influence of the wedge structure, the high-stress zone on the pipeline surface propagates faster forward than backward, resulting in the stress distribution, as presented in Figure 8(b) (C). The main sealing area is the high-stress area on the pipeline surface. Among them, the front end has the highest average stress value, which is also the most advanced area to ensure the seal.
In Figure 8(c), when the axial assembly position of ferrule changes, the development trend of the stress distribution on the ferrule surface is identical to that of the pipeline surface. Compared with the pressure-free condition, the expansion rate of the high-stress zone on the ferrule surface increases by $5.1 \%$, and the peak value increases by $7.2 \%$ when the fluid pressure is $21 \mathrm{MPa}$. During the process of assembling the ferrule from the initial position to the final position, the position of the maximum contact stress on the 
(1)

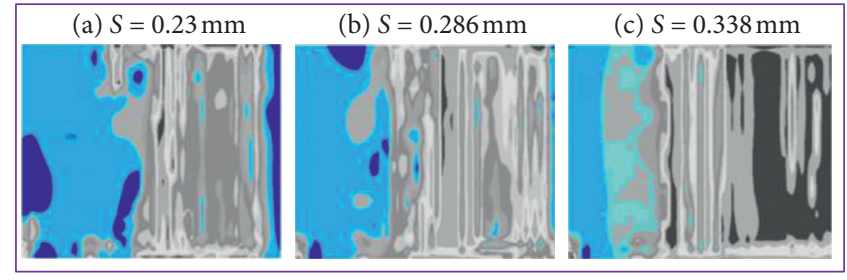

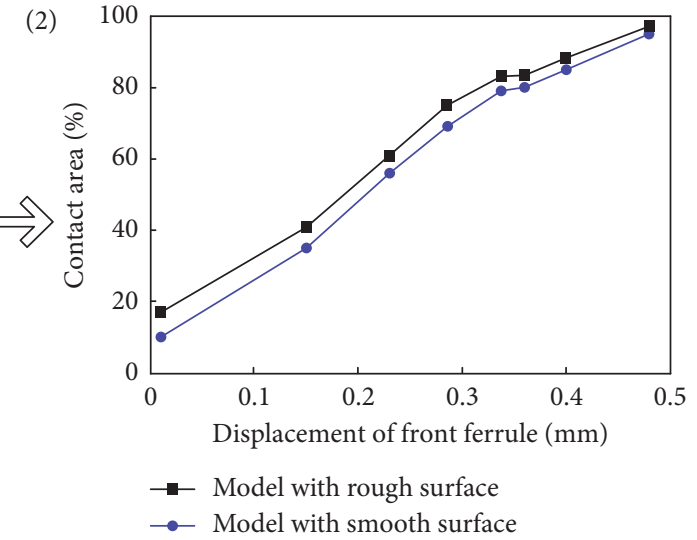

FIGURE 6: Quantitative analysis of sealing characteristics of pipeline fittings.

ferrule surface moves from the left to right. Finally, it stays at the front of the sealing area, where a favorable sealing stress state is formed. Besides, a regularly arranged strip-shaped stress distribution band is also formed in the middle and rear sections of the sealing area, which can also provide deformation energy to the main sealing area to ensure the sealing reliability.

It can be seen that the high-pressure fluid of the pipeline produces a more excellent sealing state between pipeline fittings components. Without considering the influence of the load such as vibration and temperature, the highpressure fluid can enhance the sealing contact state of pipeline fittings. The sealing state also ensures the sealing reliability of fittings during operation.

\subsection{Sealing Characteristics under High-Pressure Fluid.} The calculation of the multiscale model is employed to obtain an effective sealing contact area. That is to say, a certain degree of elastoplastic deformation between the contact materials contributes to the distribution area of the microconvex fusion. As shown in Figure 9, the corresponding relationship between the fluid pressure and effective sealing contact area is calculated according to the sealing state of the pipeline surface. It can be found that the contact area tends to increase with the improved fluid pressure, which suggests a linear correlation between them (correlation coefficient $R^{2}=0.96$ ). Within the range of fluid pressure $(7 \sim 56 \mathrm{MPa})$, the contact area percentage changes from 56 to $72 \%$, and the fluctuation range is less than $16 \%$. Therefore, the effect of high-pressure fluid has a small influence on the effective sealing contact area between pipeline and ferrule.

Based on the above calculation and analysis, it can be known that the high-pressure fluid does not significantly affect the effective sealing contact area between pipeline and ferrule. However, it can significantly increase the distribution ratio of the stress peak and the high stress of the contact surface. According to Figure 9, the area change of the highstress area on the pipeline surface with the increasing fluid pressure can be obtained. With the increased fluid pressure of pipeline, the area of the high-stress zone on the surface of pipeline rises, which exhibits a significant linear correlation (correlation coefficient $R^{2}=0.975$ ). Meanwhile, within the range of fluid pressure variation in the figure, the area of the high-stress zone on the pipeline surface changes by over $50 \%$. These results indicate that the fluid pressure greatly influences the high-stress area distribution on the contact surface between pipeline and ferrule. During the service of pipeline fittings, the high-stress area ensures the effectiveness and reliability of the fluid seal of pipeline fittings. At the same time, it is essential for the safe operation of the pipeline system. In addition, under the fluid pressure of $56 \mathrm{MPa}$, the difference between the contact area and the high-stress area is $10 \%$, indicating that almost all the sealing contact surface is under high stress.

Based on the linear fitting of data points of the contact area and high-stress area, two fitting straight lines are achieved to represent the change rule of the contact area and high-stress area. In Figure 9, two fitting lines have one intersection point at $72 \mathrm{MPa}$, demonstrating that the contact area and high-stress area are the same and all the contact zone is under high stress. When the working pressure of the pipeline system is $72 \mathrm{MPa}$, the optimal assembly displacement of ferrule is $0.23 \mathrm{~mm}$.

In summary, the fluid pressure has a greater influence on the high-stress distribution area than that of the contact area. These two areas are equal under the pressure of $72 \mathrm{MPa}$. According to the above analysis of the sealing mechanism of pipeline fittings, the high-pressure fluid can play a positive role in ensuring the sealing reliability of pipeline fittings. Moreover, when all the contact zone is under high stress, the excellent sealing characteristics can be achieved.

3.4. Influence of High-Pressure Switch. Flight control and attitude adjustment will switch the hydraulic system between the high-pressure, low-pressure, and nonpressure load states, making the aviation hydraulic pipeline system work under a high- and low-pressure alternating strong fluid load environment. This alternation of high or low pressure will influence the sealing characteristics of fittings, and leakage may occur especially under low or no pressure. Therefore, it is necessary to study the sealing state of pipeline fittings after 


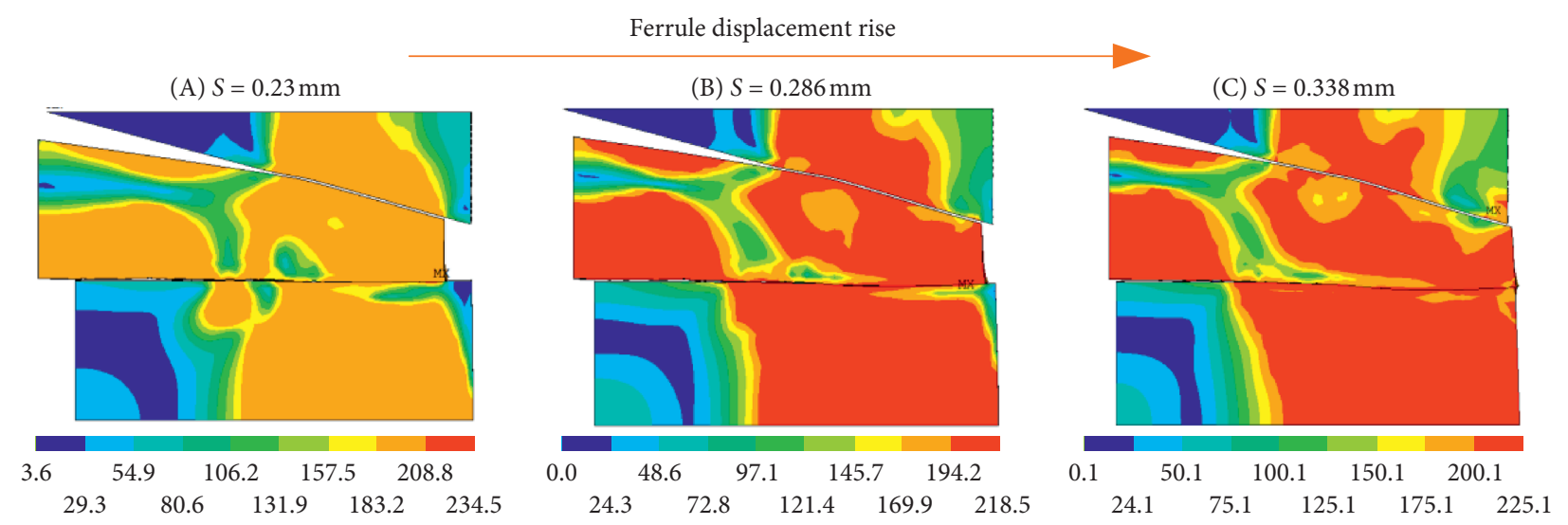

(a)

Ferrule displacement rise

(A) $P=7 \mathrm{MPa}$
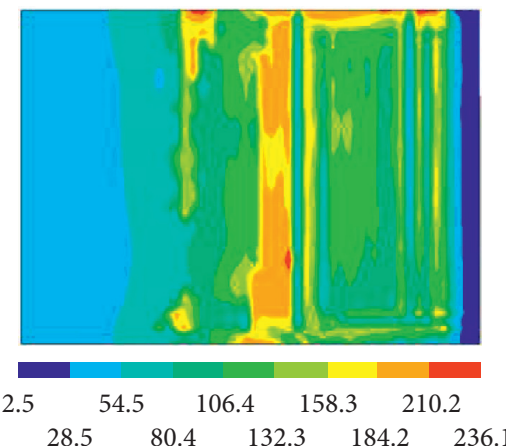

(B) $P=28 \mathrm{MPa}$

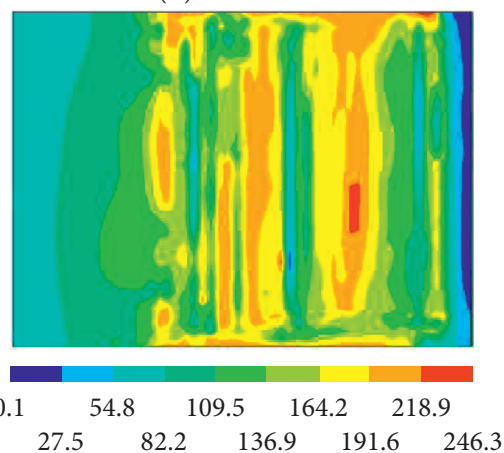

(C) $P=56 \mathrm{MPa}$

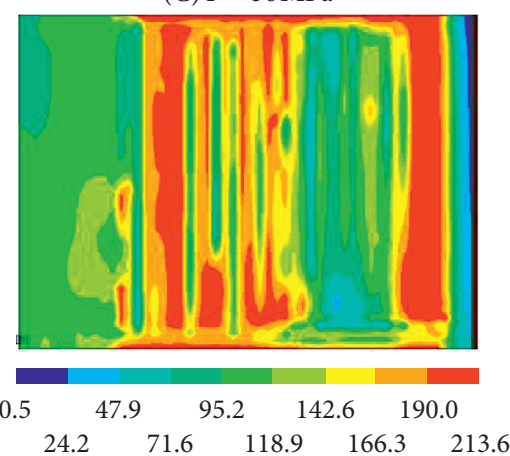

(b)

Ferrule displacement rise

(A) $P=7 \mathrm{MPa}$

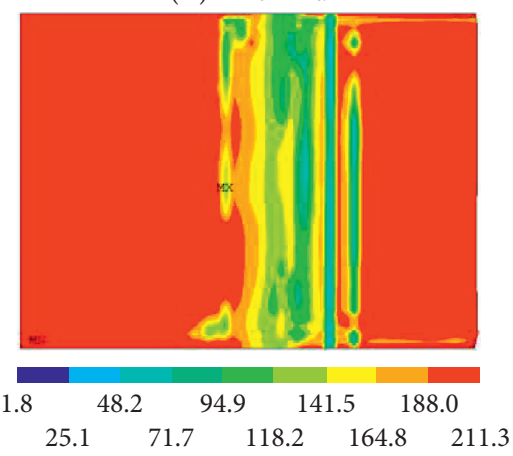

(B) $P=28 \mathrm{MPa}$

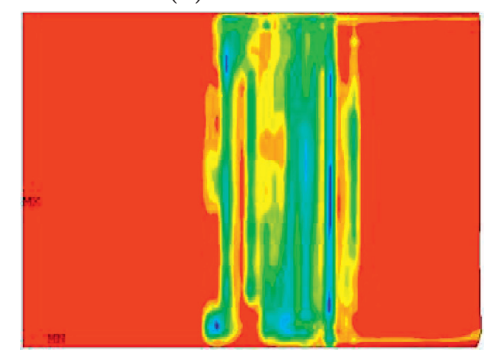

24.6

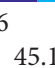

65.7
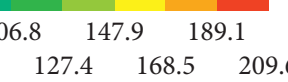

(c)

Figure 7: Stress distribution of fittings under different fluid pressures. (a) Seal state of fittings. (b) Stress distribution of pipeline surface. (c) Stress distribution of ferrule surface.

a high-pressure switch and obtain the change rule of sealing characteristics of fittings.

The elastoplastic behavior of microconvex bodies of the sealing zone under alternating high- and low-pressure loads can be described by the following formulas. The expressions of nominal strain $\varepsilon_{\text {nom }}$ and stress $\sigma_{\text {nom }}$ are expressed as follows:

$$
\begin{gathered}
\varepsilon_{\text {nom }}=\frac{\Delta l}{l_{\mathrm{o}}}, \\
\sigma_{\text {nom }}=\frac{F}{A_{\mathrm{o}}},
\end{gathered}
$$




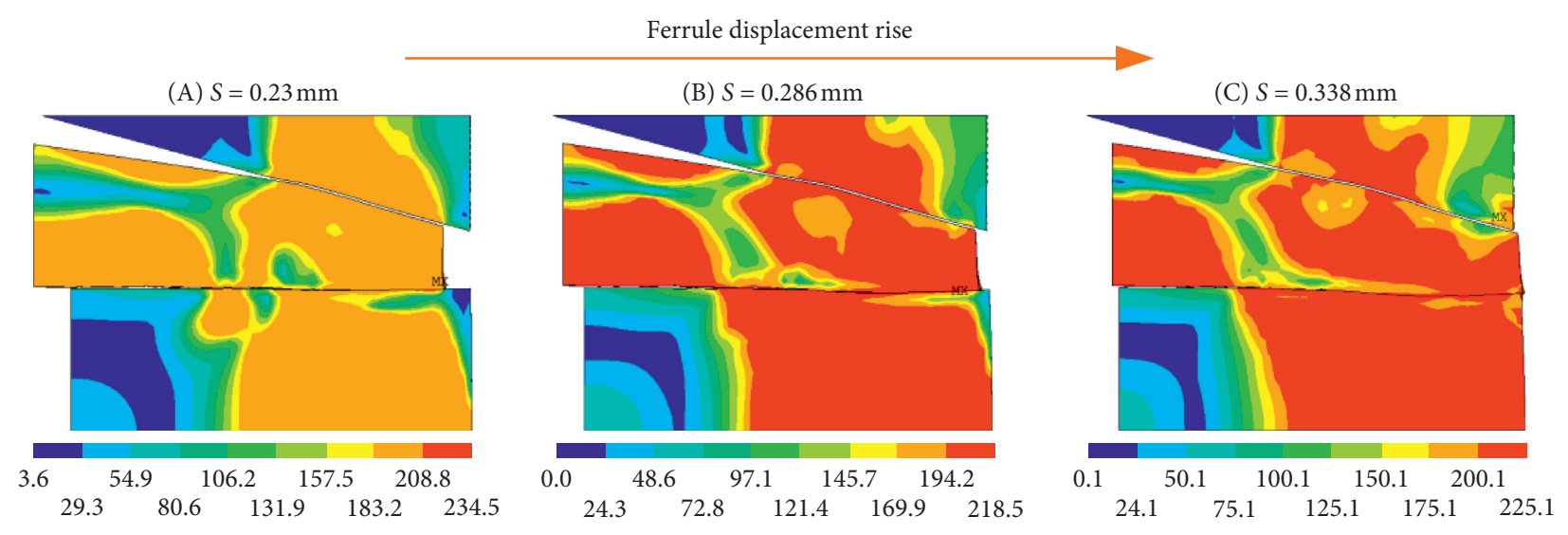

(a)

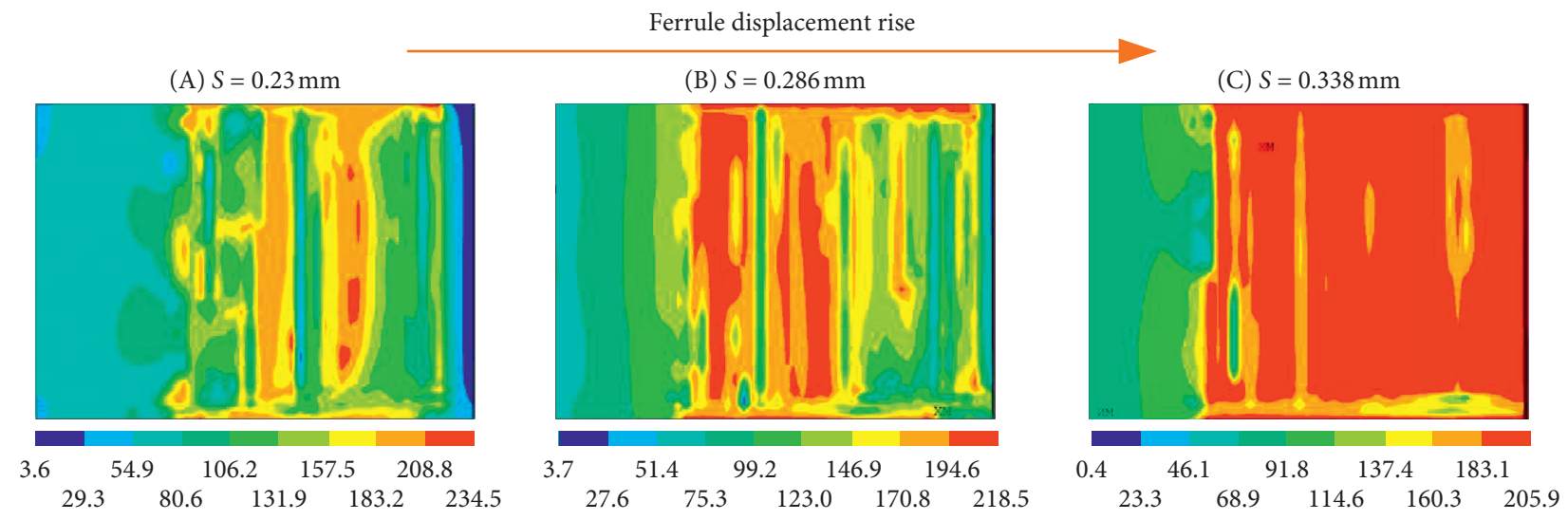

(b)

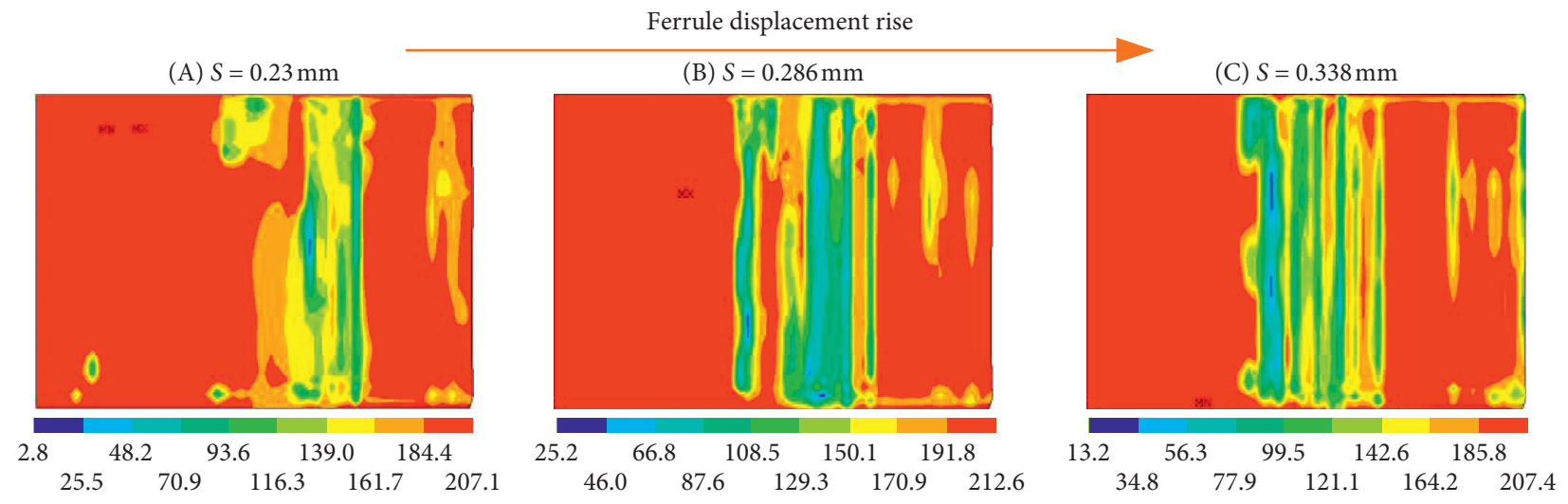

(c)

FIGURE 8: Stress distribution of fittings under different ferrule displacements and the $21 \mathrm{MPa}$ fluid pressure. (a) Seal state of fittings. (b) Stress distribution of pipeline surface. (c) Stress distribution of ferrule surface.

where $\Delta l$ refers to the specimen length variable, $l_{\mathrm{o}}$ denotes the initial length of the specimen, Frepresents the load force, and $A_{\mathrm{o}}$ is the initial load area.

To correctly depict the change of the cross-sectional area during large deformation, the relationship between true strain $\varepsilon_{\text {true }}$, true stress $\sigma_{\text {true }}$ and nominal strain $\varepsilon_{\text {nom }}$, nominal stress $\varepsilon_{\text {nom }}$ is expressed as follows:

$$
\begin{aligned}
& \varepsilon_{\text {true }}=\int_{l_{\mathrm{o}}}^{l} \frac{\mathrm{d} l}{l}=\ln \left(\frac{l}{l_{\mathrm{o}}}\right)=\ln \left(1+\varepsilon_{\mathrm{nom}}\right), \\
& \sigma_{\text {true }}=\frac{F}{A}=\frac{F}{A_{\mathrm{o}}+\left(l_{\mathrm{o}} / l\right)}=\sigma_{\mathrm{nom}}\left(1+\varepsilon_{\mathrm{nom}}\right) .
\end{aligned}
$$




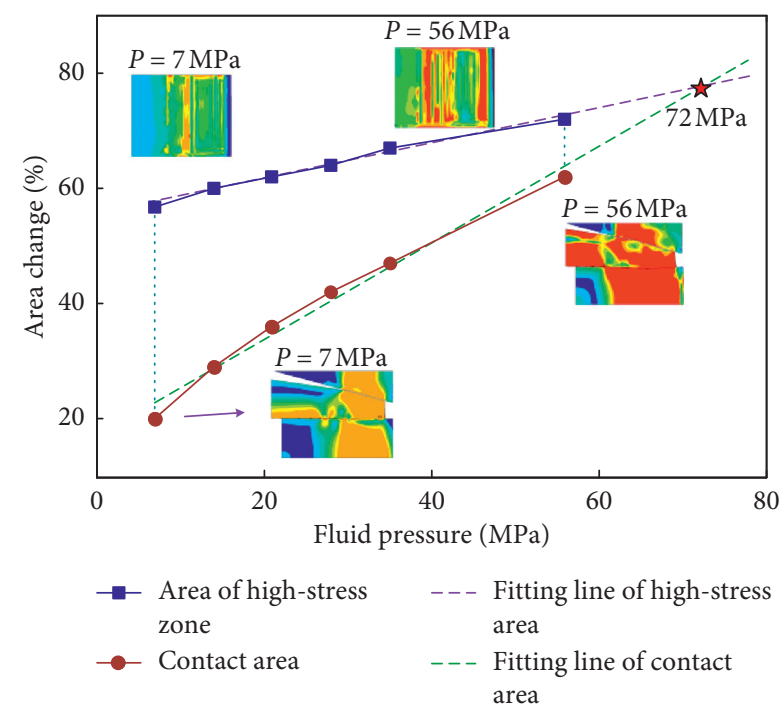

FIGURE 9: Relationship between the contact area, high-stress zone area, and different fluid pressures.

During the fitting tightening process, the strain $\varepsilon_{\text {true }}$ produced by the sealing material of fittings consists of plastic strain $\varepsilon_{\mathrm{pl}}$ and elastic strain $\varepsilon_{\mathrm{el}}$.

Due to the difference of fluid pressure, the creep of pipeline fitting material is different. Thus, three sets of pressure rise and drop simulations are carried out. During the calculation process, the fluid pressure rises from $7 \mathrm{MPa}$ to 35,56 , and $72 \mathrm{MPa}$, respectively. Then, it is gradually unloaded to $7 \mathrm{MPa}$. Based on the calculation results, the changing rules of the sealing characteristics of fittings are displayed in Figure 10. Notably, it can be seen that the contact area between the pipeline and ferrule enlarges with increased fluid pressure. When the fluid pressure is relieved, the contact area and area of the high-stress zone cannot be completely restored to the original sealing state. When the fluid pressure decreases from the high pressure to low pressure, the unrestored area enlarges with the increased maximum fluid pressure. Moreover, the area change of the high-stress zone is larger than that of the contact area.

This phenomenon can be attributed to the elastic-plastic behavior of the microconvex bodies of sealing surfaces of fittings. When the deformation of the metal material is small, the material basically exhibits linear elastic behavior. However, the yield stress of the material will increase, and the work hardening phenomenon will occur. When the fluid pressure in the pipeline is removed, the structure of the pipeline wall is restored to an unpressurized state, and the resilience force is almost equal to the pressure at the time of pressing. However, because this force cannot restore the already hardened material, there is a contact state change between sealing surfaces. In addition, due to larger stress and deformation during the pressure switching, larger plastic deformation occurs in the microconvex bodies of the high-stress area, which leads to a larger area change compared with the sealing area.

3.5. Assembly Method considering Fluid Pressure. The good contact between metal and metal is the prerequisite for effective sealing. After the assembly of pipeline fittings, the

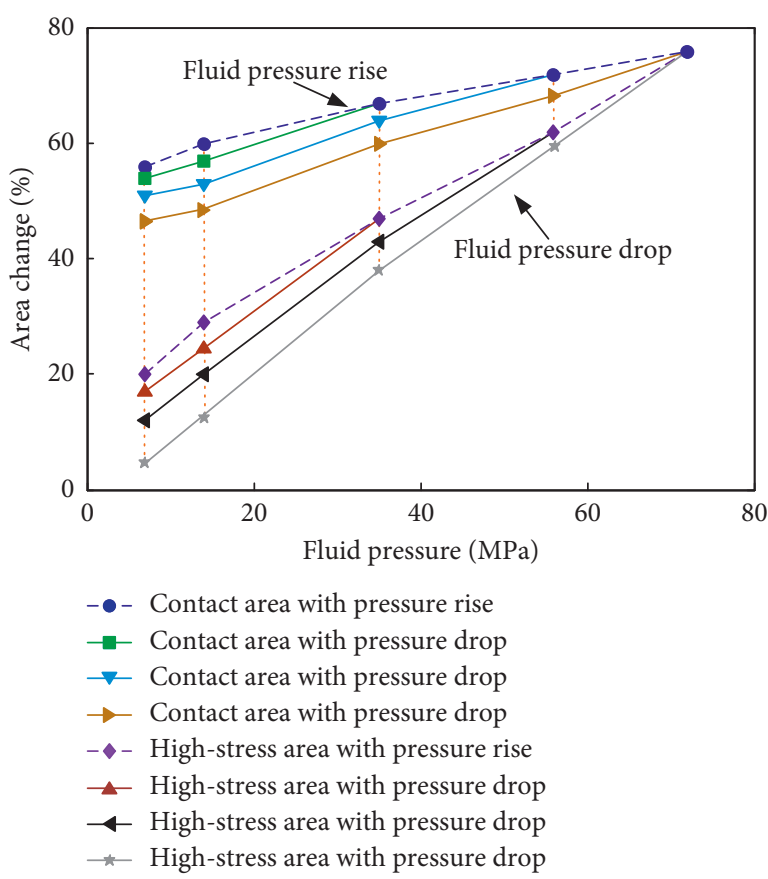

FIgURE 10: Contact area and high-stress zone area with the increased and decreased fluid pressure.

microconvex bodies of the sealing area are under a certain contact state. During the service period, the high-pressure fluid will change the contact state between the microconvex bodies of the sealing area of pipeline fittings. In the sealing area, the high-pressure fluid will make the microconvex bodies contact more closely, and the high-stress area will be formed. That is to say, the convex bodies produce elasticplastic deformation, and the effective contact area between the two surfaces increases, thus further ensuring the effective seal between the metals. However, due to the load variation, the repeated elastic-plastic deformation of the microconvex bodies leads to the creep of the material, causing the decrease of the contact tightness between the microconvex bodies and the decrease of the sealing characteristics of pipeline fittings. Therefore, the pipeline fittings under action of high-pressure fluid need reasonable and scientific assembly methods to ensure the stability of the sealing characteristics of pipeline fittings.

The sealing characteristics of pipeline fittings is affected by the manufacturing process. The assembly method is another important influencing factor. Under different assembly positions and the same working fluid pressure, the sealing characteristics of fittings are calculated to obtain the optimal assembly position of the ferrule. To show the calculation process of the assembly method, $21 \mathrm{MPa}$ fluid pressure is applied as the maximum working pressure to calculate the optimal assembly position of the ferrule.

Through calculating the sealing state of fittings in Figure 8 , the relationship between the displacement of the ferrule and contact area can be investigated in Figure 11. The diagram shows that the effective sealing contact area increases with the axial assembly position of the ferrule, which indicates a linear change law. Due to the influence 


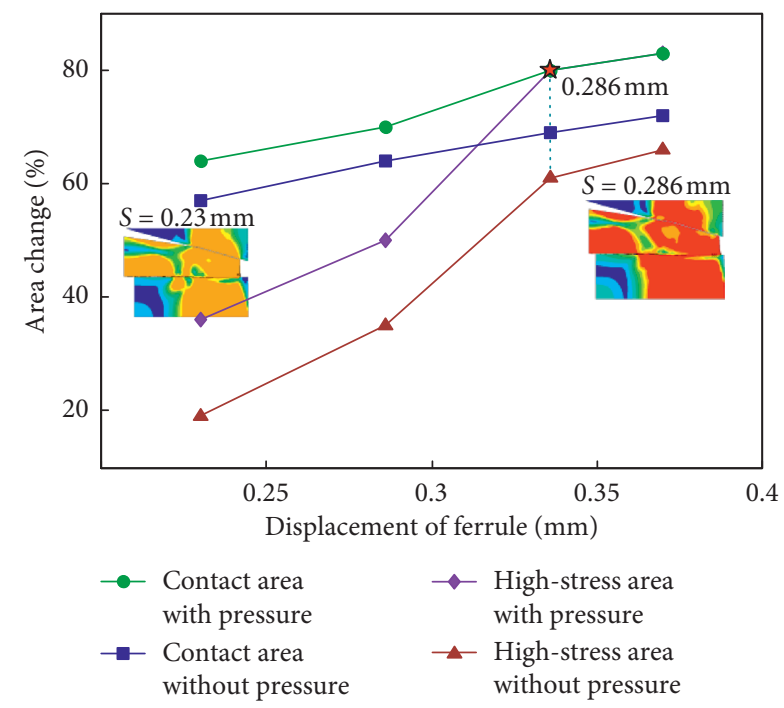

FIgURE 11: Contact area and high-stress area under different assembly positions.

of fluid pressure, the contact area increases by $13 \%$. In addition, when the ferrule is in the buckling deformation state $(S \approx 0.29-0.335 \mathrm{~mm})$, the slope of the curve slightly rises due to the influence of fluid pressure. When the buckling state disappears, the slope immediately returns to the original value. With the increased fluid pressure, the high-stress area continues to increase, and the variation trend of the relation curve is equivalent to the dispersion of the contact area in Figure 11. However, compared with the range of area change, the fluid pressure exerts a great influence on the high-stress area of the sealing area. Moreover, the contact area is equal to the high-stress area at the displacement of $0.286 \mathrm{~mm}$, which is a reasonable assembly position of the ferrule under fluid pressure of $21 \mathrm{MPa}$. In comparison with the calculation in Figure 11, the reasonable assembly position of the ferrule is $0.23 \mathrm{~mm}$ under $72 \mathrm{MPa}$ fluid pressure. In short, with the increased fluid pressure, the optimal displacement of the ferrule will decrease to achieve the excellent sealing characteristics.

Combined with the analysis of the contact state of pipeline fitting sealing area, the microcontact state of sealing joint surface, and the quantitative analysis of the sealing characteristics, the influence law of high-pressure fluid on the sealing characteristics of pipeline fittings is clearly revealed. At the same time, the phenomenon of "high pressure no leakage and low pressure leakage" of pipeline fittings is expounded, and the assembly method of pipeline fittings under the action of the high-pressure fluid is also proposed. Through the calculation and analysis, the creep is the main reason for the change of the sealing characteristics of pipeline fittings. The creep of the material caused by the change of the fluid load is inevitable. Therefore, the creep of pipeline fittings material can only be restrained by more scientific and reasonable assembly method and pipeline fitting material matching, so as to ensure the reliability of the sealing characteristics of pipeline fittings during the service period.

\section{Conclusion}

This study aims at investigating the influence of the highpressure fluid on pipeline fittings and the high-pressure fluid switching. The conclusions are shown as follows:

(1) According to the stress distribution of sealing surfaces, the multiscale model could more accurately reflect the change in the sealing area compared with the model with smooth surfaces. The high-stress zone could optimize microconvex bodies of the sealing surface during the assembly and improve the sealing reliability under the fluid pressure.

(2) The fluid pressure could boost the sealing effectiveness and reliability of pipeline fittings. When the fluid pressure increases, both the area of contact and high-stress zone increase, while the fluctuation range of the contact area is small. Meanwhile, the highstress area considerably varies. In addition, when the pressure is $72 \mathrm{MPa}$, the contact area is equal to the area of the high-stress zone, which is an excellent sealing state.

(3) Under the action of high-pressure fluid, when the high fluid pressure is removed, both the area of contact and high-stress zone cannot be completely restored to the original area. The difference in the area increases with the fluid pressure, and the difference in the high zone is larger than that of the contact area. This can be explained that due to larger stress and deformation during the pressure switching, larger plastic deformation occurs in the microconvex bodies of high-stress areas, leading to more area change compared with the sealing area.

(4) The sealing characteristics of fittings are linear under the influence of the constant pressure fluid and various tightening positions. By comparing the sealing characteristics of fittings under different conditions with and without fluid pressure, it can be concluded that the high-pressure fluid can improve the sealing characteristics of fittings under different assembly displacements, and the high-stress area significantly increases. Moreover, the displacement of 0.286 and $0.23 \mathrm{~mm}$ is a reasonable assembly position of the ferrule for 21 and $72 \mathrm{MPa}$ fluid pressure. With the increased fluid pressure, the optimal assembly displacement of the ferrule will be reduced for achieving the excellent sealing characteristics.

\section{Nomenclature}

$A_{\mathrm{o}}: \quad$ Initial load area

E: Modulus of elasticity

F: $\quad$ Load force

$l_{\mathrm{o}}: \quad$ Initial length of the specimen

$\Delta l$ : Specimen length variable

$R^{2}$ : Correlation coefficient

$S: \quad$ Displacement

$\varepsilon_{\text {nom }}:$ Nominal strain

$\varepsilon_{\text {true }}:$ True strain 


$$
\begin{array}{ll}
\varepsilon_{\mathrm{el}}: & \text { Elastic strain } \\
\varepsilon_{\mathrm{pl}}: & \text { Plastic strain } \\
\sigma_{\text {nom }}: & \text { Nominal stress } \\
\sigma_{\text {true }}: & \text { True stress } \\
\mu: & \text { Poisson's ratio } \\
\rho: & \text { Density. }
\end{array}
$$

\section{Data Availability}

All data used to support the findings of the study are available within the article.

\section{Conflicts of Interest}

The authors declare that they have no conflicts of interest to the present study, and they do not have any commercial or associative interest standing for conflicts of interest associated with the work submitted.

\section{Acknowledgments}

This research was financially supported by the Facility Horticulture Laboratory of Universities in Shandong Program (nos. 2018YY057 and 2018YY004).

\section{References}

[1] C. Lu, S. Wang, and X. Wang, "A multi-source information fusion fault diagnosis for aviation hydraulic pump based on the new evidence similarity distance," Aerospace Science and Technology, vol. 71, pp. 392-401, 2017.

[2] M. S. Xia, "Ferrule fittings and its application," Hydraulic, Pneumatic and Sealing, no. 2, pp. 46-47, 2000.

[3] M. Mihsein and M. D. Seymour, "Intermixing twin ferrule tube fittings-is it safe?" Journal of Engineering Design, vol. 10, no. 4, pp. 391-416, 1999.

[4] B. Yan, N. Y. Sun, and A. P. Liu, "Sealing principle and leakage prevention analysis of 24 degree ferrule fittings," Modern Manufacturing Technology and Equipment, no. 4, p. 64, 2015.

[5] S. X. Li, K. S. Huang, J. J. Cai, Q. X. Zhang, and T. H. Fu, "Study on contact state and pressure of medium pressure mechanical seals," Lubrication Engineering, vol. 41, no. 1, pp. 10-14, 2016.

[6] R. Metcalfe, C. A. Kittmer, and G. W. Brown, "Effects of pressure and temperature changes on end-face seal characteristics," ASLE Transactions, vol. 25, no. 3, pp. 361-371, 1981.

[7] K. Willenborg, S. Kim, and S. Wittig, "Effects of reynolds number and pressure ratio on leakage loss and heat transfer in a stepped labyrinth seal," Journal of Turbomachinery, vol. 123, no. 4, pp. 815-822, 2001.

[8] J. Li, X. Yan, G. J. Li et al., "Effects of pressure ratio and sealing clearance on leakage flow characteristics in the rotating honeycomb labyrinth seal," in Proceedings of the ASME Turbo Expo 2007: Power for Land, Sea, and Air, pp. 1199-1206, 2007.

[9] J. Gibson, K. Thole, J. Christophel et al., "Effects of the main gas path pressure field on rim seal flows in a stationary linear cascade," in Proceedings of the ASME Turbo Expo 2015: Turbine Technical Conference and Exposition, Montreal, Canada, June 2015.

[10] S. Kucharski and G. Starzynski, "Study of contact of rough surfaces: modeling and experiment," Wear, vol. 311, no. 12, pp. 167-179, 2014.
[11] G. Zhao, S.-X. Li, Z.-L. Xiong, W.-D. Gao, and Q.-K. Han, “A statistical model of elastic-plastic contact between rough surfaces," Transactions of the Canadian Society for Mechanical Engineering, vol. 43, no. 1, pp. 38-46, 2019.

[12] B. An, X. Wang, Y. Xu, and R. L. Jackson, "Deterministic elastic-plastic modelling of rough surface contact including spectral interpolation and comparison to theoretical models," Tribology International, vol. 135, pp. 246-258, 2019.

[13] J. Guo, P. He, Z. Liu, and H. Huang, "Investigation of an improved planar revolute clearance joint contact model with rough surface," Tribology International, vol. 134, pp. 385-393, 2019.

[14] P. Wagner, P. Wriggers, L. Veltmaat, H. Clasen, C. Prange, and B. Wies, "Numerical multiscale modelling and experimental validation of low speed rubber friction on rough road surfaces including hysteretic and adhesive effects," Tribology International, vol. 111, pp. 243-253, 2017.

[15] Y. Waddad, V. Magnier, P. Dufrénoy, and G. De Saxcé, “A multiscale method for frictionless contact mechanics of rough surfaces," Tribology International, vol. 96, pp. 109-121, 2016.

[16] K. Poulios and P. Klit, "Implementation and applications of a finite-element model for the contact between rough surfaces," Wear, vol. 303, no. 1-2, pp. 1-8, 2013.

[17] D. K. Karupannasamy, M. B. de Rooij, and D. J. Schipper, "Multi-scale friction modelling for rough contacts under sliding conditions," Wear, vol. 308, no. 1-2, pp. 222-231, 2013.

[18] S. Belghith, S. Mezlini, H. BelhadjSalah, and J.-L. Ligier, "Modeling of contact between rough surfaces using homogenisation technique," Comptes Rendus Mécanique, vol. 338, no. 1, pp. 48-61, 2010.

[19] J. F. Wenk, L. S. Stephens, S. B. Lattime, and D. Weatherly, "A multi-scale finite element contact model using measured surface roughness for a radial lip seal," Tribology International, vol. 97, pp. 288-301, 2016.

[20] F. Pérez-Ràfols, R. Larsson, and A. Almqvist, "Modelling of leakage on metal-to-metal seals," Tribology International, vol. 94, pp. 421-427, 2016.

[21] X. Jia, S. Jung, W. Haas, and R. F. Salant, "Numerical simulation and experimental study of shaft pumping by plunge ground shafts with rotary lip seals," Tribology International, vol. 48, pp. 155-161, 2012.

[22] F. Zhang, J. Liu, X. Ding et al., "An approach to calculate leak channels and leak rates between metallic seal surfaces," Journal of Tribology, vol. 139, no. 1, Article ID 011708, 2017.

[23] A. Thatte and R. F. Salant, "Effects of multi-scale viscoelasticity of polymers on high-pressure, high-frequency sealing dynamics," Tribology International, vol. 52, pp. 75-86, 2012.

[24] C. Marie, D. Lasseux, H. Zahouani et al., "An integrated approach to characterize liquid leakage through metal contact seal," European Journal of Mechanical \& Environmental Engineering, vol. 48, no. 2, pp. 81-86, 2003.

[25] Y. Yan, J. Zhai, P. Gao, and Q. Han, "A multi-scale finite element contact model for seal and assembly of twin ferrule pipeline fittings," Tribology International, vol. 125, no. 57, pp. 100-109, 2018. 